\title{
Netrin-1 and Semaphorin 3A Promote or Inhibit Cortical Axon Branching, Respectively, by Reorganization of the Cytoskeleton
}

\author{
Erik W. Dent, ${ }^{1}$ Aileen M. Barnes, ${ }^{1}$ Fangjun Tang, ${ }^{2}$ and Katherine Kalil ${ }^{1,2}$ \\ ${ }^{1}$ Department of Anatomy and ${ }^{2}$ Neuroscience Training Program, University of Wisconsin, Madison, Wisconsin 53706
}

\begin{abstract}
In many CNS pathways, target innervation occurs by axon branching rather than extension of the primary growth cone into targets. To investigate mechanisms of branch formation, we studied the effects of attractive and inhibitory guidance cues on cortical axon branching. We found that netrin-1, which attracts cortical axons, and FGF-2 increased branching by $>50 \%$, whereas semaphorin $3 \mathrm{~A}$ (Sema3A), which repels cortical axons, inhibited branching by $50 \%$. Importantly, none of the factors affected axon length significantly. The increase in branching by FGF- 2 and the inhibition of branching by Sema3A were mediated by opposing effects on the growth cone (expansion vs collapse) and on the cytoskeleton. FGF-2 increased actin polymerization and formation of microtubule loops in growth cones over many hours, whereas Sema3A depolymerized actin filaments, attenuated microtubule dynamics, and collapsed microtubule arrays within minutes. Netrin-1 promoted rapid axon branching, often without involving the growth cone. Branches formed de novo on the axon shaft within $30 \mathrm{~min}$ after local application of netrin-1, which induced rapid accumulation of actin filaments in filopodia. Importantly, increased actin polymerization and microtubule dynamics were necessary for axon branching to occur. Taken together, these results show that guidance factors influence the organization and dynamics of the cytoskeleton at the growth cone and the axon shaft to promote or inhibit axon branching. Independent of axon outgrowth, axon branching in response to guidance cues can occur over different time courses by different cellular mechanisms.
\end{abstract}

Key words: actin; microtubule; netrin-1; semaphorin 3A; axon branch; cortical development

\section{Introduction}

During development, efferent cortical axons navigate over long pathways such as the corpus callosum and the corticospinal tract by responses of their primary growth cones to attractive and inhibitory guidance cues. Innervation of cortical and spinal targets occurs, however, by interstitial axon branching rather than extension of the primary growth cone into targets (O'Leary et al., 1990; Halloran and Kalil, 1994; Kuang and Kalil, 1994). Thus, branching is an important form of axon guidance (Kennedy and Tessier-Lavigne, 1995), and there is accumulating evidence that factors that attract or repel growth cones during pathfinding can also promote or inhibit branching of their axons. For example, semaphorin 3A (Sema3A), a member of a large family of guidance cues (Castellani and Rougon, 2002; Pasterkamp and Kolodkin, 2003), repels cortical axons in situ and in vitro (Bagnard et al., 1998; Polleux et al., 1998, 2000) and appears to reduce cortical axon branching (Bagnard et al., 1998; Bagri et al., 2003). On the

Received Nov. 5, 2003; revised Jan. 9, 2004; accepted Feb. 2, 2004

This work was supported by National Institutes of Health Grant NS14428 to K.K. Movies of several figures can be viewed at http://kalil.anatomy.wisc.edu.

Correspondence should be addressed to Dr. Katherine Kalil, Department of Anatomy, 1300 University Avenue, University of Wisconsin, Madison, WI 53706. E-mail: kakali@@wisc.edu.

E. W. Dent's present address: Department of Biology, Massachusetts Institute of Technology, Building 68-270, 77 Massachusetts Avenue, Cambridge, MA 02139.

DOI:10.1523/JNEUROSCI.4963-03.2004

Copyright $\odot 2004$ Society for Neuroscience $\quad$ 0270-6474/04/243002-11\$15.00/0 other hand, Sema3A repels the growth cones of retinal neurons but promotes branching of their axons after the growth cones recover from collapse (Campbell et al., 2001). Netrins promote outgrowth of a wide variety of axons (Manitt and Kennedy, 2002) and in explant cocultures have been shown to attract growing cortical axons (Metin et al., 1997; Richards et al., 1997); however, their effects on axon branching are not known.

Previous studies have shown that axon branching is mechanistically linked to behaviors of the growth cone (Kalil et al., 2000; Dent et al., 2003). Axons of retinal ganglion neurons extend filopodia and lamellipodia after growth cone collapse (Davenport et al., 1999), and the primary growth cones of cortical axons enlarge during prolonged pausing behaviors to leave remnants behind on the axon shaft after the axon resumes forward extension (Szebenyi et al., 1998). These filopodial and lamellipodial remnants subsequently give rise to interstitial branches. FGF-2 was found to increase cortical axon branching by inducing growth cone arrest and enlargement (Szebenyi et al., 2001), but it is not known whether effects of other guidance factors on axon branching are also mediated by changes in growth cone behaviors and morphologies. Directed growth from the growth cone and the axon shaft is also mechanistically linked by a similar dynamic reorganization of the cytoskeleton in which actin-microtubule interactions are an essential component (Gallo and Letourneau, 1998, 1999; Dent et al., 1999; Dent and Kalil, 2001). The effects of physiological guidance cues on cytoskeletal dynamics in verte- 
brate neurons have been little studied. We hypothesized that guidance cues would promote or inhibit branching by opposing changes in the organization of the cytoskeleton both at the growth cone and at branch points on the axon shaft. In the present study we sought to test this hypothesis first by comparing the effects of attractive and inhibitory guidance cues on cortical growth cones and development of axon branches and second by analyzing dynamic changes in the cytoskeleton induced by these guidance cues.

\section{Materials and Methods}

Cell culture. Cultures were prepared from the sensorimotor cortex obtained from embryonic day (E) 14 and postnatal day (P) 0-3 golden Syrian hamsters (Mesocricetus auratus) as described previously (Dent and Kalil, 2003). Hamsters are born after a $16 \mathrm{~d}$ gestation period (E16 = $\mathrm{P} 0)$, causing them to be more premature than other rodents at birth (mice, 19-20 d gestation; rats, 21-22 d gestation). Cortical neurons remained viable for 5-7 d under these culture conditions, with a large number of pyramidal neurons and a small number of glial cells $(<5 \%)$. For FGF-2 and Sema3A experiments, neurons were cultured on 0.5 $\mathrm{mg} / \mathrm{ml}$ poly-D-lysine coated with $20 \mu \mathrm{g} / \mathrm{ml}$ laminin. Laminin was excluded in netrin-1-treated cultures because it is known to switch netrin1-induced attraction to repulsion (Hopker et al., 1999).

Preparation of Sema3A supernatants. To prepare Sema3A supernatants, we used a stable cell line of human embryonic kidney (HEK) 293 cells that produces alkaline phosphatase (AP)-tagged Sema3A (gift from Dr. Mary Halloran, University of Madison, Madison, WI). Cell transfection was performed with standard procedures using Lipofectamine 2000. To concentrate the Sema3A supernatant, we centrifuged the supernatant through a concentrating filter (Millipore, Bedford, MA) that selectively retains high molecular weight proteins $>100 \mathrm{kDa}$. To obtain estimates of Sema3A concentrations, we used a colorimetric assay (Sigma, St. Louis, $\mathrm{MO})$. Control cultures were treated with supernatant from APtransfected HEK cells. FGF-2 was obtained from Promega (Madison, $\mathrm{WI}$ ), and netrin-1 was from R \& D Systems (Minneapolis, MN).

Immunocytochemistry. All cultures were fixed in $4 \%$ paraformaldehyde (EM Sciences) in Krebs' buffer with 0.4 m sucrose (Dent and Meiri, 1992) to preserve the cytoskeleton and fine morphology of growth cones. To determine whether cortical neurons at late embryonic and early postnatal ages express receptors to Sema3A, we labeled the cultures with antibodies to the plexin-A1 (1:50) (Santa Cruz Biotechnology, Santa Cruz, CA) and neuropilin-1 receptors (1:100) (gift from Hajime Fujisawa, Nagoya University, Nagoya, Japan). To determine whether cortical neurons at P0 express receptors to netrin-1, we labeled cultures with antibodies to the deleted in colorectal cancer (DCC) receptors $(4 \mu \mathrm{g} / \mathrm{ml})$ (Oncogene). All fluorescently conjugated secondary antibodies were used at 1:200 (Jackson ImmunoResearch, West Grove, PA).

Long-term imaging. Imaging was performed as described previously (Szebenyi et al., 1998, 2001). Briefly, the culture dishes were sealed to prevent $\mathrm{pH}$ changes and transferred to the heated stage of a Zeiss $35 \mathrm{M}$ inverted microscope (Carl Zeiss, Thornwood, NY). Cortical neurons were viewed with a $20 \times / 0.5$ numerical aperture (NA) Neofluor objective under phase-contrast optics and imaged with a MicroMax cooled CCD digital camera (Princeton Instruments, Trenton, NJ) controlled by a Metamorph-based digital imaging system (Universal Imaging, West Chester, PA). For some experiments, neurons were followed in timelapse over the course of several days by using the etchings on the coverslips as landmarks. Other experiments involved imaging neurons at several time points, during time periods up to $96 \mathrm{hr}$ after plating.

Quantitation of axon branches after bath application of guidance factors. Supernatants of Sema3A, netrin-1, and FGF-2 were bath applied to cultures of cortical neurons from embryonic and 1-3 d hamster sensorimotor cortex. After various time periods, branches along cortical axons were counted under phase microscopy. For quantification of branch length, branch number and axon length images of fixed cells were acquired with a $20 \times / 0.5 \mathrm{NA}$ Neofluor phase-contrast objective. Axon lengths were measured from the cell body to the distal extent of the central region of the growth cone. An axon was defined as the process that was at least double the length of the next longest minor process. For determination of axon versus branch, the axon was defined as the process that remained parallel to the axon segment proximal to the branch point. Branches were defined as processes extending at orthogonal angles to the axon. These counts were performed blind. We normalized the numbers of branches as a function of branches per $100 \mu \mathrm{m}$ of axon length and adopted the criterion that a branch must be at least $20 \mu \mathrm{m}$ long, as described previously (Dent and Kalil, 2001).

Local application of netrin-1. A stock solution of netrin-1 $(25 \mu \mathrm{g} / \mathrm{ml})$ was locally applied through a $0.5 \mu \mathrm{m}$ pipette attached to an Eppendorf microinjection system. The pipette was positioned $20 \mu \mathrm{m}$ from a cortical axon not in contact with any other processes, and netrin- 1 was ejected repeatedly from the tip every $2 \mathrm{sec}$ for 30-60 min. Images of the axon were acquired in DIC every $50 \mathrm{sec}$.

Injection of fluorescent probes. Microtubules were labeled by injection of rhodamine-tubulin (Cytoskeleton Inc.). Actin filaments were labeled by injection of Alexa488-phalloidin (Molecular Probes, Eugene, OR). Alexa488-phalloidin was stored at $-20^{\circ} \mathrm{C}$ as a $6.6 \mu \mathrm{M}$ methanol stock. For injections, $200 \mu \mathrm{l}$ of phalloidin was dried under nitrogen and resuspended in $0.5 \mu \mathrm{l}$ of dry DMSO. Rhodamine-tubulin was diluted to 2-4 $\mathrm{mg} / \mathrm{ml}$ in injection buffer (100 mM PIPES, $0.5 \mathrm{~mm} \mathrm{MgCl}_{2}, \mathrm{pH} 6.9$ ), and 9.5 $\mu \mathrm{l}$ was added to the phalloidin-DMSO mixture, resulting in final concentrations of $132 \mu \mathrm{M}$ Alexa488-phalloidin and 1.9-3.8 mg/ml TMRtubulin in the injection pipette. Injection of the phalloidin-tubulin mixture and preparation of injected neurons for live cell imaging was performed as described previously (Dent and Kalil, 2001).

Time-lapse fluorescence imaging. Dual fluorophore live cell imaging was performed by projecting images of fluorescently labeled cells through a Keller port of a Nikon TE300 Quantum inverted microscope equipped with dual excitation/emission filter wheels (Sutter Instruments) and a Princeton Instruments MicroMax 512BFT cooled CCD camera containing a back-thinned, frame transfer EEV CCD57-10 chip (Roper Scientific) (Dent and Kalil, 2003). Neurons were imaged in time lapse (10 sec intervals) with a $100 \times / 1.4$ NA PlanApo CFI60 objective (Nikon). This objective is chromatically corrected so that the Alexa488phalloidin-labeled actin filaments and TMR-labeled microtubules could be imaged without adjusting focus. All peripherals were controlled by Metamorph. Images were collected and saved in 16-bit format.

Image processing and data analysis. All measurements were taken from 16-bit images. Measurements of levels of staining in fixed samples and levels of fluorescent compounds in living cells were made on background-subtracted 16-bit images. For measurement of splayed microtubules and F-actin bundles, images were processed with the noneighbors deconvolution function in Metamorph before measuring the length and counting the number of microtubules. Deconvolution of the images allowed for the unambiguous identification of microtubules over background noise. All multicolor images were merged with the overlay images function in Metamorph. To determine the dynamic relationship between F-actin and microtubules in living growth cones and branches, movies of montaged images were constructed and analyzed frame by frame. Movies of montaged images were compiled from time-lapse images of F-actin; images of microtubules and merged images of microtubule-F-actin were compiled with the montage stacks function in Metamorph and saved as individual tiff images. Images presented in the figures were sharpened with the no-neighbors deconvolution function in Metamorph and compiled as 8-bit gray scale or 24-bit color images (Adobe Photoshop). Graphs were constructed in Microsoft Excel, and statistical analyses were performed with SPSS (Jandel Software).

\section{Results}

In these experiments we examined the effects of attractive and inhibitory guidance cues on the branching of cortical axons. We chose for study neurons with obvious pyramidal morphologies that form the majority of cortical neurons and give rise to long branched efferent axons. Neurons were plated at low density, and only neurons not in contact with other cells were included in our measurements. We used neurons in cultures ranging from late embryonic $(\mathrm{E} 14$, where E16 = P0) to early postnatal $(\mathrm{P} 0-\mathrm{P} 3)$ 

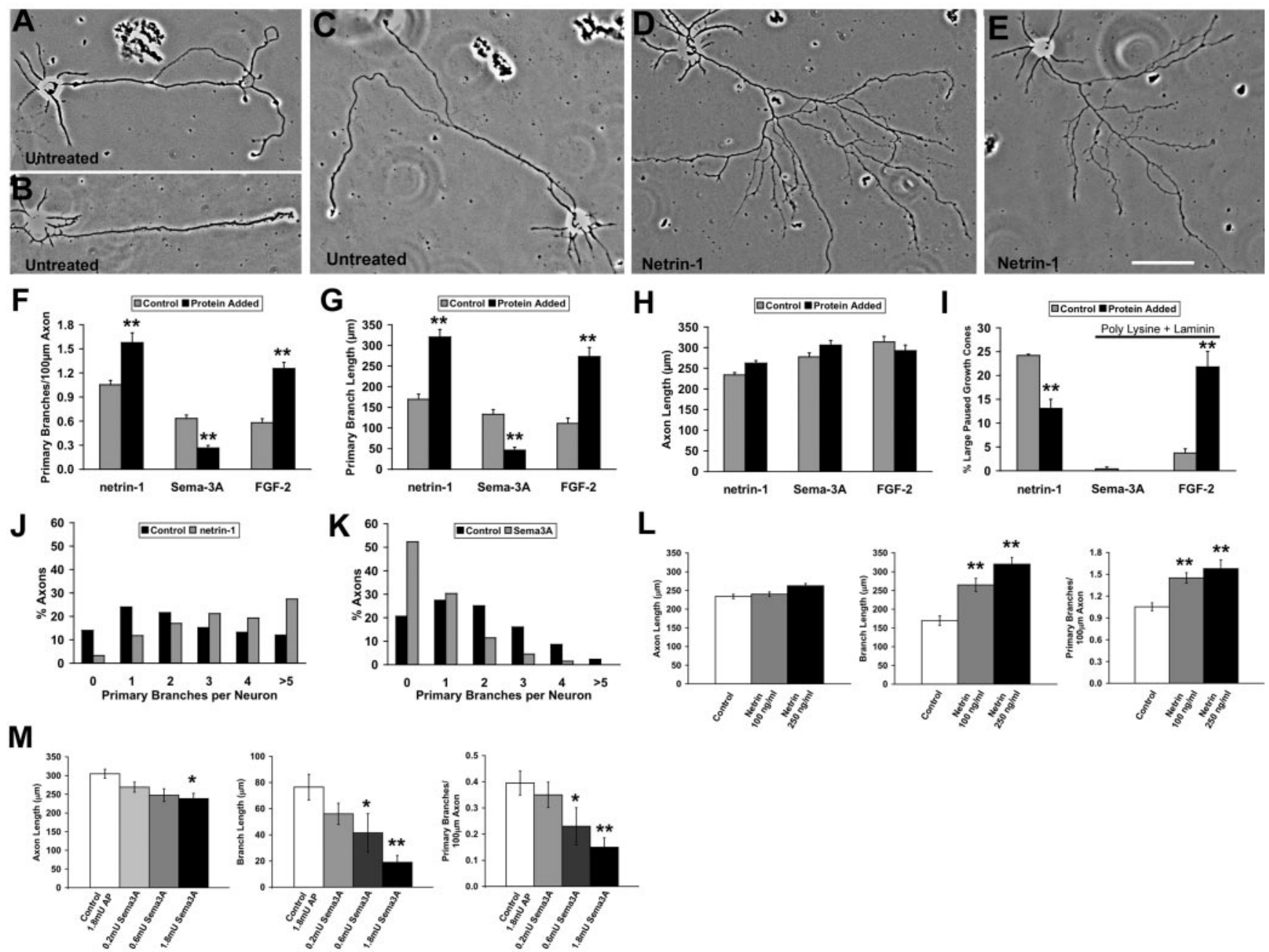

Figure 1. Netrin-1 and FGF-2 increase and Sema3A decreases axon branching without affecting axon outgrowth. A-C, Examples of control neurons (untreated) after $72 \mathrm{hr}$ in culture. $D, E$, Examples of cortical neurons treated with netrin- $1(250 \mathrm{ng} / \mathrm{ml})$ for $72 \mathrm{hr}$. F, G, Bar graphs showing that treatment $(72 \mathrm{hr})$ with netrin- $1(250 \mathrm{ng} / \mathrm{ml})$ and FGF- $2(10 \mathrm{ng} / \mathrm{ml})$ increases axon branching and branch length, whereas Sema3A (supernatant) decreases axon branching and branch length (all treatments: ${ }^{* *} p<0.001$ for all conditions; Student's $t$ test; $n>150$ neurons; separate controls used for each treatment group). $H$, Bar graphs showing that netrin-1, FGF-2, and Sema3A treatment has no effect on axon length (all treatments: ${ }^{*} p<0.05 ;$ Student's $t$ test; $n>150$ neurons). I, Bar graph showing that netrin-1 decreases the percentage of large paused growth cones in the cultures, whereas FGF- 2 increases the percentage $(* * p<0.001$; Student's $t$ test). There are virtually no large paused growth cones after Sema3A treatment. All error bars are \pm SEM. J, K, Histograms showing that netrin- 1 increases the percentage of axons with large numbers of branches, whereas Sema3A increases the percentage of axons with few or no branches. L, M, Dose-response experiments with netrin-1 and Sema 3 A showing that increasing concentrations of each factor increases or decreases axon branch length and number, respectively; however, there is little effect on axon length ( ${ }^{*} p<0.05$ and ${ }^{* *} p<0.01$; ANOVA with Dunn's post hoc tests). All experiments were performed on E14-P3 cultures. Scale bar: $A-E, 20 \mu \mathrm{m}$.

hamster cortex. At these ages axons develop interstitial branches similar in numbers and time course to those in vivo (Szebenyi et al., 1998). Because neurons in different cortical layers are born and differentiate at different times, our cultures represent a heterogeneous mixture of different cell types at different stages of development.

\section{Effects of netrin-1 on cortical axon branching}

Netrin-1 is a diffusible guidance factor that attracts efferent cortical axons (Serafini et al., 1996; Metin et al., 1997; Richards et al., 1997). To determine whether netrin-1 also influences cortical axon branching, we bath applied netrin-1 at concentrations of $100-250 \mathrm{ng} / \mathrm{ml}$ to dissociated P0 cortical cultures for periods up to $72 \mathrm{hr}$ (Fig. 1A-E). These concentrations are slightly below the optimal concentration $(400 \mathrm{ng} / \mathrm{ml})$ for outgrowth from cortical explants (Metin et al., 1997) and are similar to other published studies in dissociated cell culture on the effects of netrin-1 (de la Torre et al., 1997; Forcet et al., 2002). In netrin-1 and matching control experiments, we excluded laminin from the substrate because it is known to convert the effects of netrin- 1 from attraction to repulsion in growth cones of frog retinal ganglion cells (Hopker et al., 1999). In untreated control cultures, axons averaged 2.3 primary branches $(n=250)$ after $72 \mathrm{hr}$, whereas neurons treated with $250 \mathrm{ng} / \mathrm{ml}$ netrin-1 for $72 \mathrm{hr}$ averaged 3.67 branches per axon $(n=212)$, an increase of $\sim 57 \%$ (Fig. $1 A-E)$. We normalized these values as numbers of primary branches per $100 \mu \mathrm{m}$ of axon (Fig. $1 F$ ); however, not all neurons were affected equally. Approximately 15\% of the netrin-1-treated axons had zero to one branch compared with $38 \%$ for controls, and $27 \%$ had five or more branches compared with $12 \%$ for controls (Fig. $1 J$ ). Some netrin-1-treated axons had 10 or more branches, which was never seen in control cultures. Thus, for neurons that responded to netrin-1, the increase in branching was actually more than twofold. We also found a clear dose-response effect (Fig. 1L) such that the total number of branches increased by $43 \%$ at $100 \mathrm{ng} / \mathrm{ml}$ and $57 \%$ at $250 \mathrm{ng} / \mathrm{ml}$ ( $n=426$ axons). At these two concentra- 

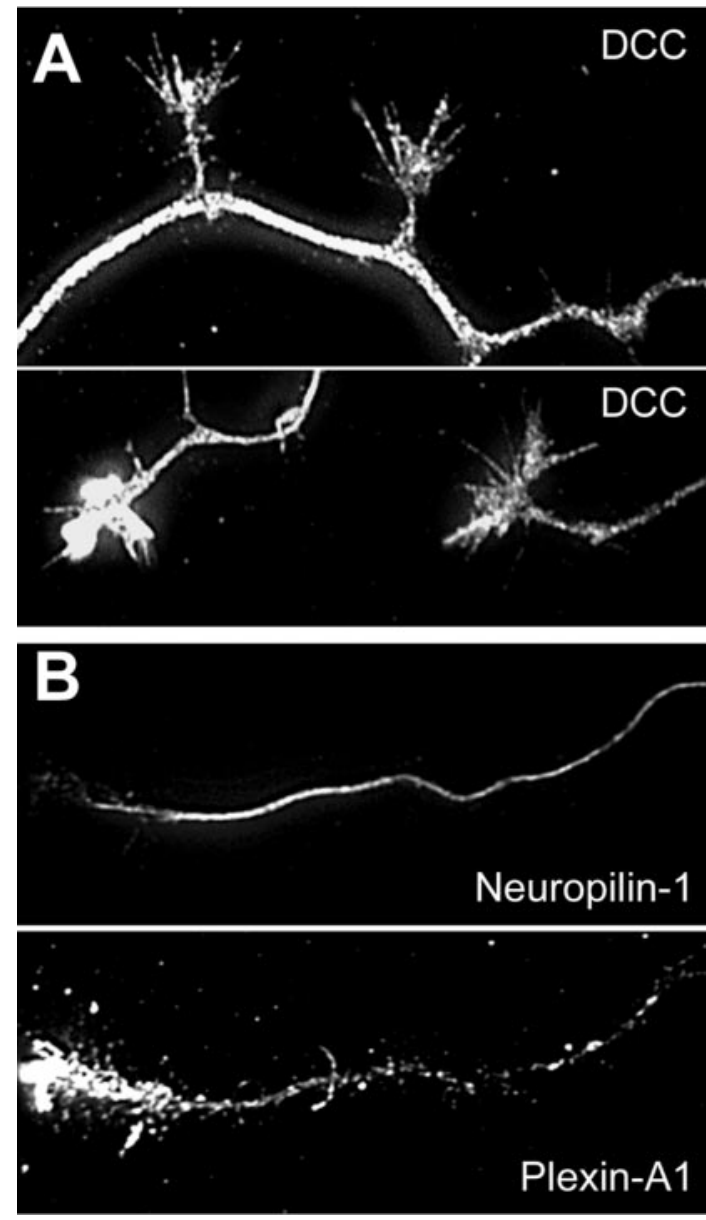

Figure 2. Receptors for Sema3A and netrin-1 are distributed along axons of cortical neurons. $A$, Two examples of $\mathrm{PO}$ cortical neurons labeled with antibodies to the netrin-1 receptor DCC. Note the even punctate distribution of DCC along the axon shaft, in newly formed branches (top panel), and in growth cones (bottom panel). B, An individual E14 cortical neuron labeled with antibodies to neuropilin-1 and plexin-A1. Note the even distribution of the Sema3A receptor neuropilin-1 and the distal concentration of the Sema $3 A$ receptor plexin-A1 on the axon and growth cone. Scale bar, $10 \mu \mathrm{m}$.

tions of netrin-1, branch length was also increased by 56 and $89 \%$, respectively, which included an increase in the number of branches that had developed secondary and tertiary branches; however, length of the primary axons was not affected (Fig. $1 H, L)$. As a positive control, we compared these results with those from cultures treated with FGF-2, which uniformly increased axon branching by approximately threefold (Fig. $1 F, G$ ) in agreement with Szebenyi et al. (2001), but also did not increase axon length significantly (Fig. $1 H$ ). Thus, both netrin-1 and FGF-2 increased the number and length of axon branches but did not affect primary axon length.

Previous studies have shown that netrin-1 is present in the ventral telencephalon at appropriate times in development to guide growing axons (Serafini et al., 1996; Metin et al., 1997; Braisted et al., 2000). To verify that cortical neurons express netrin-1 receptors at the age when their axons respond to this guidance factor, we immunostained cultures from $\mathrm{P} 0$ cortex with antibodies to the DCC receptor that is known to mediate the attractant effects of netrin-1 (de la Torre et al., 1997). As shown in Figure $2 A$, we found that DCC receptors were densely and uniformly distributed along all processes of cortical neurons. Thus at this age, cortical neurons are capable of responding to netrin-1.
Previous observations showed that the increased axon branching after FGF-2 treatment resulted from an increase in large paused growth cones that, over periods of $48-72 \mathrm{hr}$, gave rise to axon branches (Fig. 1I) (Szebenyi et al., 2001). Netrin-1 application, however, actually decreased the number of large paused growth cones from $24 \%$ of the growth cones in controls to $16 \%$ (at $100 \mathrm{ng} / \mathrm{ml}$ ) and 12\% (at $250 \mathrm{ng} / \mathrm{ml}$ ) (Fig. 1I). Thus, it seemed unlikely that increased branching after netrin-1 treatment originated from large paused growth cones. Therefore, to visualize how axon branches developed, we performed timelapse imaging of netrin-1-treated neurons $(n=3)$ at 1 min intervals for up to $12 \mathrm{hr}$. Remarkably, within $4 \mathrm{hr}$ one of the axons had already developed several branches $\sim 100 \mu \mathrm{m}$ long (Fig. $3 A$ ), which did not occur in controls until $48 \mathrm{hr}$ in culture. Although some of the branches developed from expanded branch points along the axon shaft, many branches extended de novo (67\%) from smooth regions of the axon, which occurred infrequently in controls (20\%) (Szebenyi et al., 1998). Branches from netrin-1treated neurons began as filopodia, which were either transient or developed highly motile growth cones that grew rapidly (averaging $10-20 \mu \mathrm{m} / \mathrm{hr}$ ) to form a stable branch within a few hours. Thus, the effect of netrin-1, in contrast to FGF-2, was an accelerated and increased formation of branches that often developed directly from the axon shaft.

\section{Localized effects of netrin-1 on axon branching}

Gradients of guidance cues and neurotrophic factors induce localized directional changes in growth cones and their axons (Ming et al., 2002). Locally applied FGF-2-coated beads induced the formation of cortical axon branches within $10 \mu \mathrm{m}$ of the bead (Szebenyi et al., 2001). To determine whether netrins could locally induce new axonal growth, we positioned glass pipettes 20 $\mu \mathrm{m}$ from the shafts of cortical axons $(n=5)$ and repeatedly pulsed netrin-1 $(25 \mu \mathrm{g} / \mathrm{ml})$ from the pipette for 30-60 min. In one example shown in Figure $4 A$, the pipette was positioned close to an expanded axon branch point. Within $30 \mathrm{~min}$, new processes extended from the branch point and grew $11 \mu \mathrm{m}$ toward the source of netrin-1. In addition, new secondary branches extended de novo from smooth regions of an existing branch. In contrast, regions of the axon farther away from the pipette tip showed no new growth. In other neurons (Fig. $4 B$ ), axonal processes extended de novo from smooth regions of the axon shaft in the vicinity of the pipette but not from axon regions farther away. These results show that local application of netrin-1 can promote localized directed axon branching within minutes as opposed to the protracted extension of branches over several days after application of FGF-2-coated beads.

\section{Effects of Sema3A on axon branching}

Semaphorin 3A has been shown in slice overlay cultures to orient efferent cortical axons away from the cortical plate by chemorepulsion (Polleux et al., 1998, 2000). It has also been reported that application of Sema3A decreased cortical axon branching by 50\% (Bagnard et al., 1998); however, these branching assays were performed on cortical explants grown on membranes from Sema3A-producing cells. Because axons in this culture system grew out at high density and often fasciculated with one another, it was not possible to determine whether there was a specific inhibition in axon branching or a general decrease in axon outgrowth, which included a diminution of branches. Furthermore, effects of axon-axon contact may have influenced axon branch- 
ing (Davenport et al., 1999). Therefore, we considered it important to measure changes in axon branching in low-density cortical cultures in which the origin and lengths of branches could be determined unambiguously. We first bath applied Sema3A at increasing concentrations to cultures from $\mathrm{P} 1$ and $\mathrm{P} 3$ sensorimotor cortex. Sema3A reduced axon branching by $12-70 \%$ at $\mathrm{P} 1$ and by $12-50 \%$ at $\mathrm{P} 3(n=$ 12-58 neurons at each concentration) compared with controls treated with supernatant from HEK cells transfected with the AP vector alone. In all further experiments we used the concentration of Sema3A that reduced branching by 50\% without compromising the health of the cultures. Although we did not determine the absolute concentration of Sema3A, we did perform a colorimetric AP assay to determine the units of AP in all experiments and matched the AP activity in all control (HEK 293 cells transfected with the AP vector alone) experiments. Exposure of E14 and P3 cultures to Sema3A for $72 \mathrm{hr}$ resulted in $51 \%$ fewer axon branches $(n=$ 152 axons) in comparison with controls $(n=233)$ (Fig. $1 F)$. Approximately half of the Sema3A-treated axons had no branches (Fig. $1 \mathrm{~K}$ ). Sema3A also significantly reduced branch length to $58-68 \%$ of controls at $72 \mathrm{hr}$ for E14 and P3 neurons, respectively (Fig. 1G). Surprisingly, axon length was not affected by Sema3A (Fig. $1 H$ ). We found a pronounced dose-response effect of Sema3A on the number and length of axon branches, but axon length was only slightly affected (Fig. 1M). Thus, Sema3A specifically affected branching but had no effect on axon outgrowth per se.

Next we assessed the effects of Sema3A on growth cone morphologies. In control cultures we found that at any given time $\sim 2 \%$ of the growth cones had large expanded morphologies (Fig. $1 I$ ). This is less than the $24 \%$ observed in the control experiments for netrin-1 in which omission of laminin (poly-D-lysine alone) produced a more adhesive substrate conducive to growth cone pausing and expansion. In cultures treated with Sema3A for 48 or $72 \mathrm{hr}$, we found no large growth cones. At shorter time periods, Sema3A had profound effects on growth cone morphologies. After $1 \mathrm{hr}, 50 \%$ of growth cones had collapsed morphologies, and at higher concentrations $77 \%$ of the growth cones had collapsed compared with $11 \%$ for controls ( $n=568$ for all conditions). Collapsed growth cones typically had stick-like axon tips or were condensed into a stubby ending completely lacking lamellipodia or filopodia (data not shown). Observations of growth cones fixed after 5, 15, or 30 min of Sema3A treatment showed that most of the growth cone collapse occurred within 5 min of exposure to Sema3A.

Previous studies have shown that Sema3A and its receptors, neuropilin and plexin, are present in the developing cortex and that the repellent effects of Sema3A on cortical axons are mediated by these receptors (Bagnard et al., 1998; Polleux et al., 1998). We immunostained cultures from E14 (Fig. 2B) and P3 cortex (data not shown) and found that neuropilin receptors were uniformly distributed on the cell body and all processes of the neurons, whereas plexin staining appeared to be concentrated most
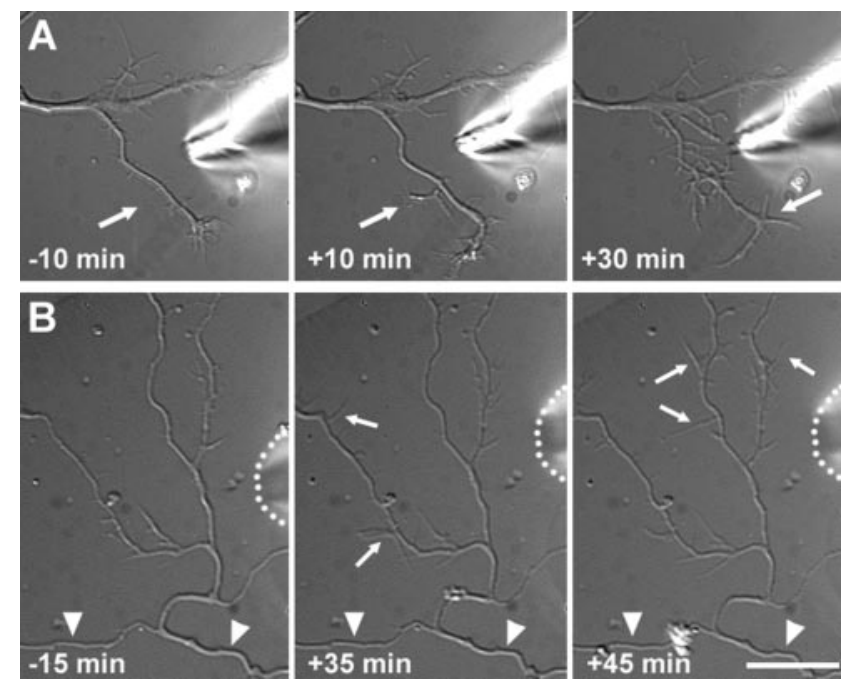

Figure 4. Local application of netrin-1 to cortical axons induces rapid filopodial protrusions localized near the pipette tip. A, A micropipette containing $25 \mu \mathrm{g} / \mathrm{ml}$ netrin- 1 is positioned near an axon that has branched. Within 10 min after the start of the pressure ejection of netrin-1, filopodia begin to sprout and elongate (arrow at $+10 \mathrm{~min}$ ). After $30 \mathrm{~min}$ of netrin- 1 application, numerous filopodia have formed, and most are elongating toward the pipette tip. Note also that the growth cone has stopped elongating and begins to branch (arrow at $+30 \mathrm{~min}$ ). $B$, Another example of filopodial formation and elongation after application of netrin-1. In this example, filopodia (arrows) form to the left of the pipette tip but not on axon regions to the top or bottom of the image (arrowheads). Note that when the pipette is moved toward the top of the image at the +35 min time point, filopodial protrusions sprout from the more distal regions of the axon, in the vicinity of the pipette tip (arrows at $+45 \mathrm{~min}$ ). Both neurons were from P1 cortical cultures. Scale bar, $20 \mu \mathrm{m}$.

heavily in growth cones and the distal region of the axon. Because almost all of the cortical neurons stained for both of these receptors, we concluded that at these ages cortical neurons are capable of responding to Sema3A. 

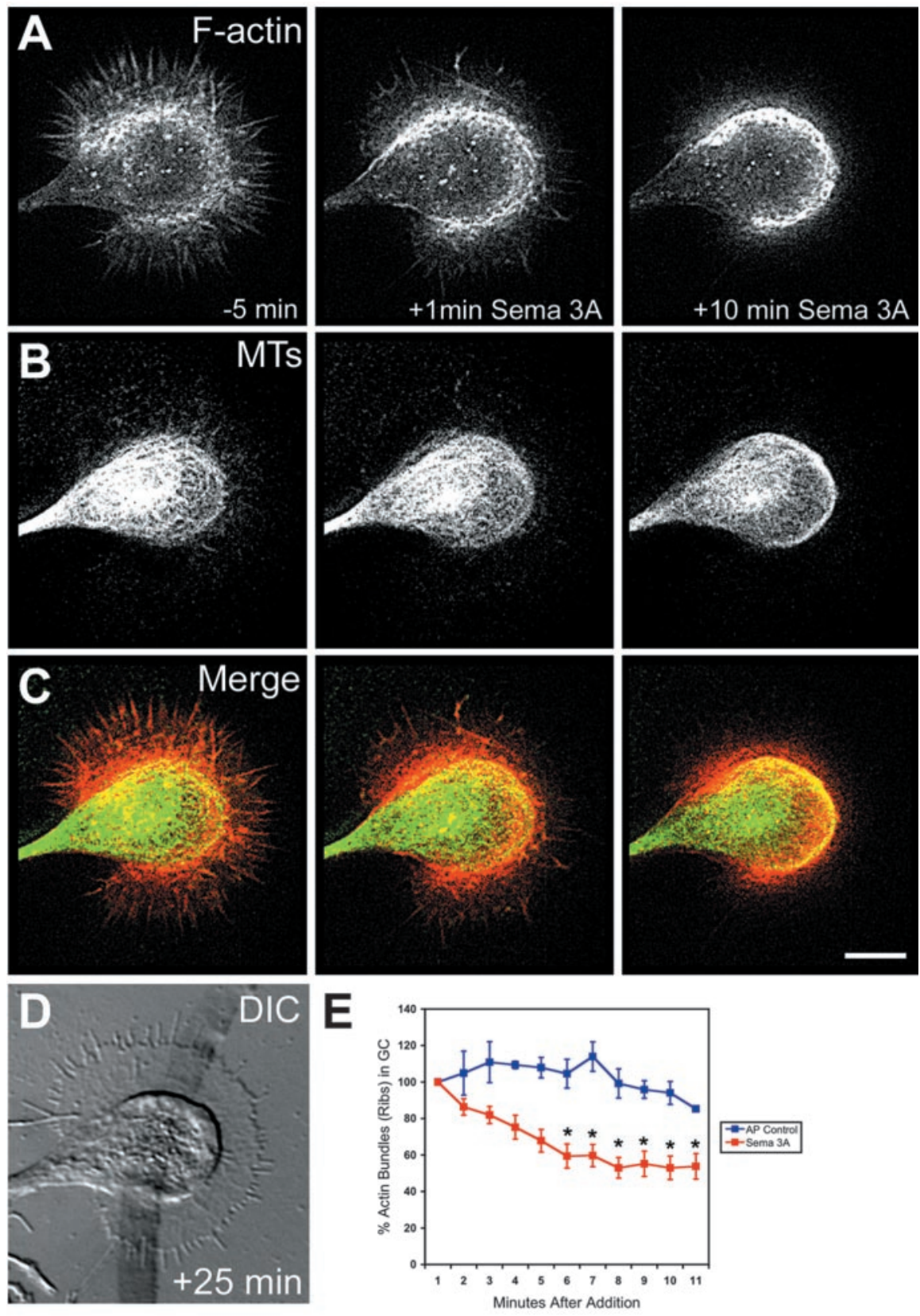

Figure 5. Sema $3 A$ rapidly decreases the number of $\mathrm{F}$-actin bundles and dynamic microtubules in the peripheral region of the growth cone without collapse of the growth cone. $A, B$, Images of a living large paused growth cone from a P3 cortical neuron coinjected with Alexa488 phalloidin to label F-actin and with rhodamine tubulin to label microtubules (MTs). Within 1 min after application of Sema3A, many of the actin bundles in the growth cone periphery disappear, concomitant with the loss of dynamic microtubules extending from the central microtubule loop. By $10 \mathrm{~min}$ after Sema3A application, the intensity of an F-actin ring surrounding the peripheral microtubule loop increases. $C$, Merged images of the same growth cone in $A$ and $B$, where F-actin is pseudocolored red and microtubules are green. D, A DIC image of the same growth cone in $A-C$ showing that the filopodia and broad lamellar region have not retracted. $E$, Graph plotting the number of actin bundles over time. Semaphorin addition resulted in a significant decrease in actin bundles over time compared with supernatant collected from AP vector-infected cells $\left({ }^{*} p<0.05\right.$; paired $t$ test). All error bars are \pm SEM. Scale bar, $10 \mu \mathrm{m}$.

\section{Effects of guidance cues on cytoskeletal reorganization}

In previous studies of cytoskeletal changes underlying the development of cortical axon branches (Dent et al., 1999; Dent and Kalil, 2001), we found that at the growth cone and at branch points along the axon shaft the reorganization of microtubules as well as interactions between dynamic microtubules and actin filaments were necessary for branch formation. To determine how guidance factors influence the cytoskeleton to inhibit or promote axon branching, we used a combination of live cell imaging and immunostaining of fixed cultures to visualize short-term and long-term reorganization of the microtubule and actin cytoskeleton. In the first set of experiments, we imaged fluorescently labeled microtubules and actin filaments in living growth cones before and after bath application of Sema3A.

In the example shown in Figure 5, before application of Sema3A the growth cone had a large spread morphology with many actin bundles, filopodia, and a characteristic microtubule loop in the central region. Microtubules extended from this region and actively explored the lamellipodium. Bath application of Sema3A did not collapse the large pausing growth cone, probably because the lamellipodium was highly adherent to the substrate (Fig. $5 D$ ). This allowed us to observe clearly the cytoskeletal changes induced by Sema3A (movies of Fig. 5 are available at http:// kalil.anatomy.wisc.edu). Imaging for 15 min at frequent intervals (every $10 \mathrm{sec}$ ) revealed that within 1 min bundled actin filaments in filopodia were depolymerized, and this attenuated the motility and protrusion of filopodia (Fig. $5 A, C, E$ ). Over a period of $10 \mathrm{~min}$, Sema3A addition significantly reduced the number of actin bundles (ribs) in the peripheral region of the growth cone compared with controls (Fig. $5 E)$. The rapid clearing of dynamic actin filaments (actin bundles and intrapodia) from the transition region resulted in a decrease of exploratory behaviors by microtubules and their subsequent collapse onto the microtubule loop (Fig. $5 B, C$ ). After Sema3A treatment, however, retrograde actin flow continued at a rate similar to pretreatment levels and not significantly different from AP-treated controls (data not shown).

In another example (Fig. 6), we analyzed cytoskeletal reorganization in an axon with a large terminal growth cone and a collateral branch tipped by a growth cone (movies of Fig. 6 are available at http://kalil.anatomy.wisc.edu). Before application of Sema3A, the growth cone on the collateral branch contained splayed microtubules and a spread lamellipodium. The primary growth cone also contained a prominent microtubule loop and a dense concentration of actin filaments and microtubules distal to the loop. These overlapping actin filaments and microtubules were exploring the growth cone periphery and resembled the organization of the cytoskeleton when a new axon was developing from a large pausing growth cone (Dent and Kalil, 2001). Addition of Sema3A had immediate inhibitory effects on the cytoskeleton. Remarkably, within 3 min the splayed microtubules collapsed into a bundle, and within $10 \mathrm{~min}$ the microtubule loop 

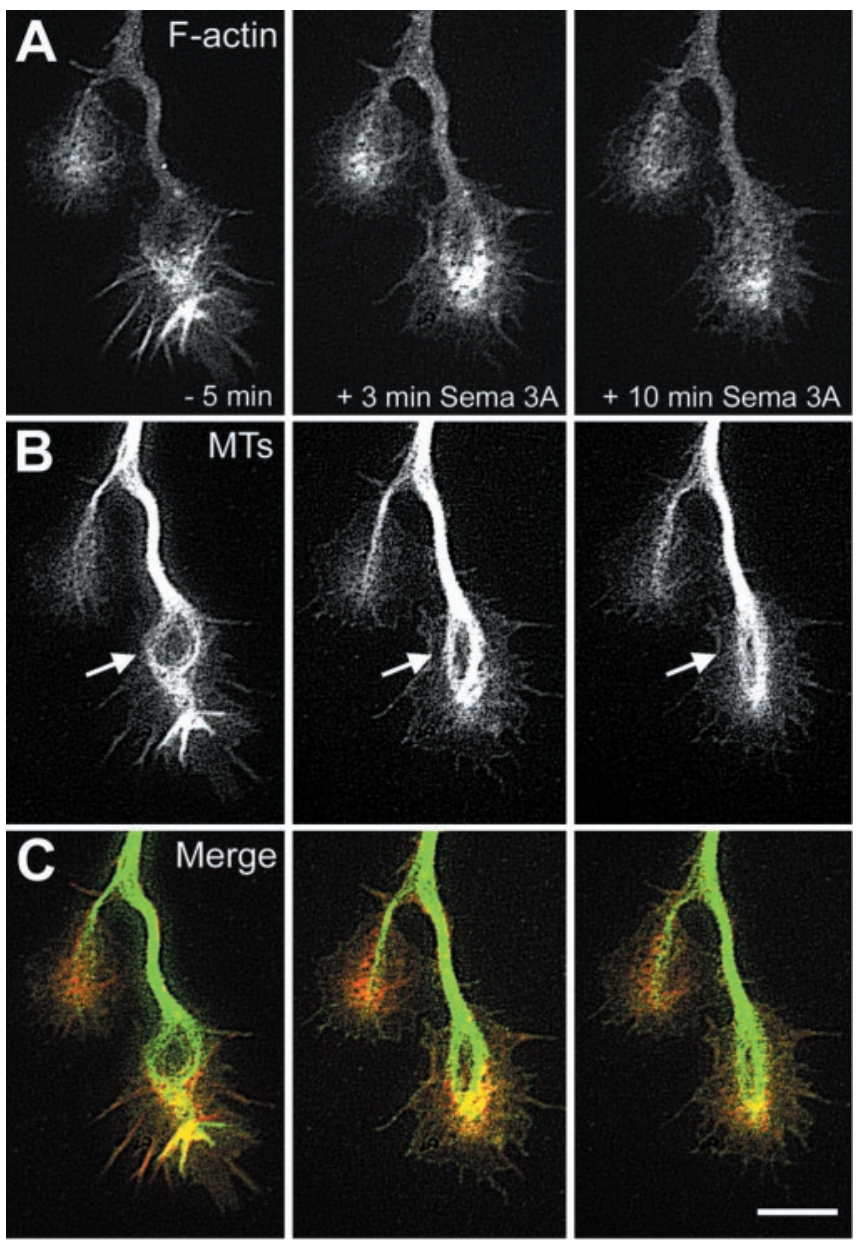

Figure 6. Sema $3 A$ collapses microtubule loops and decreases F-actin in the growth cone. $A$ $B$, Images of a living growth cone at the tip of a primary axon and a growth cone from a newly formed axon branch. This P3 cortical neuron was also coinjected with Alexa488 phalloidin to label F-actin and rhodamine tubulin to label MTs. Within 3 min after application of Sema3A, the actin bundles have disappeared, and the prominent microtubule loop in the primary growth cone has begun to collapse. Also, the splayed microtubules in the growth cone from the axon branch have collapsed into the center of the growth cone. After 10 min of Sema3A application, the microtubule loop has collapsed almost to the diameter of the axon. C, Merged images of the same growth cone in $A$ and $B$, where $\mathrm{F}$-actin is pseudocolored red and microtubules are green. Scale bar, $10 \mu \mathrm{m}$.

in the primary growth cone also collapsed into a straight bundled array. Dynamic actin filaments and microtubules ceased their exploratory movements and disappeared from the growth cone periphery, and the lamellipodia of both growth cones retracted slightly and ceased motility. Similar immediate inhibitory effects were observed on additional growth cones and developing branches $(n=17)$. Because the development of branches often depends on the maintenance of pausing regions along the axon, the depolymerization of actin filaments, the collapse of microtubule loops, and the loss of cytoskeletal dynamics by Sema3A are likely to underlie the decrease in axon branching for neurons exposed to Sema3A. In support of this view, these cytoskeletal changes resemble those that occur after application of nocodazole and latrunculin (Dent and Kalil, 2001), drugs that attenuate microtubule and actin filament dynamics, respectively. As with Sema3A, these drugs inhibit axon branching without affecting axon length.

Longer term changes in the cytoskeleton were documented previously during the development of cortical axon branches over many hours. In several cases we repeated these observations using live cell imaging at longer intervals. Observations of FGF2-treated neurons extend our previous results (Szebenyi et al., 2001) by showing that branching is accompanied by an increase in actin polymerization and a concomitant splaying of microtubules in the axon shaft and growth cone (Fig. 7A) and formation of microtubule loops in a newly formed growth cone (Fig. 7B).

Immunostaining of growth cones fixed at various stages after exposure to netrin-1 also showed a rapid and marked increase in actin filaments in the growth cone and axon shaft (Fig. 8A-E). This increase in actin filaments, often organized into distinct actin bundles, resulted in an increase in the complexity of the growth cone as determined by the formula (perimeter squared/ area) (Fig. 8F) (Bowie and Young, 1977), as has been described after application of Wnt-7a to cerebellar neurons (Hall et al., 2000). The increase in actin filament bundles and filopodia at the growth cone was accompanied by an increased number of filopodia along the axon shaft (Fig. $8 G$ ) and splaying of tyrosinated (dynamic) microtubules in growth cones (Fig. $8 A-D$ ) and axon shafts (Fig. $8 H$ ), but without an increase in large paused growth cones, as noted above. These data indicate that as opposed to a repellent guidance factor like Sema3A, attractive guidance factors such as netrin-1 and FGF-2, as well as NGF (Gallo and Letourneau, 1998), increase actin filaments, which in turn cause microtubules to splay apart or debundle. These dynamic splayed microtubules, now at orthogonal angles to the main microtubule bundle, are capable of interacting with the newly polymerized actin bundles to initiate new directions of outgrowth.

To determine whether the increase in actin filaments and microtubule dynamics, resulting in microtubule splaying, was necessary for the increase in axon branches induced by netrin-1 and FGF-2, we included latrunculin and nocodazole in the culture medium. Latrunculin decreases actin polymerization by sequestering actin monomers, which causes actin filaments to eventually depolymerize (Spector et al., 1989; Dent and Kalil, 2001). When used at nanomolar concentrations, nocodazole does not depolymerize microtubules but attenuates microtubule dynamics (Vasquez et al., 1997; Dent and Kalil, 2001). Incubation of netrin-1- and FGF-2-treated cultures with either latrunculin or nocodazole completely reversed the increase in axon branch number (Fig. 9A) and length (Fig. 9B) induced by these growth factors; however, these drugs had little effect on primary axon outgrowth (Fig. 9C) (Dent and Kalil, 2001). These data, along with those presented above, demonstrate that the increase in actin filaments and in splaying of dynamic microtubules is necessary for netrin-1- and FGF-2-induced axon branching of cortical neurons.

\section{Discussion}

In this study we show that netrin-1, the guidance cue known to attract cortical growth cones, also promotes axon branching, whereas another factor, Sema3A, repellent to cortical axons, inhibits axon branching. Importantly, none of the factors that influenced axon branching had any significant effect on axon length. Thus, netrin-1 increased branch length and Sema3A decreased branch length without affecting axon length. The growth-promoting effects of FGF-2 and the inhibitory effects of Sema3A on branching were both mediated through the growth cone, which formed expanded branch points along the axon shaft. At the growth cone and at axon branch points, FGF-2 increased long-term axon branching by inducing polymerization of actin filaments and formation of microtubule loops over many hours. Conversely, Sema3A inhibited axon branching by depoly- 


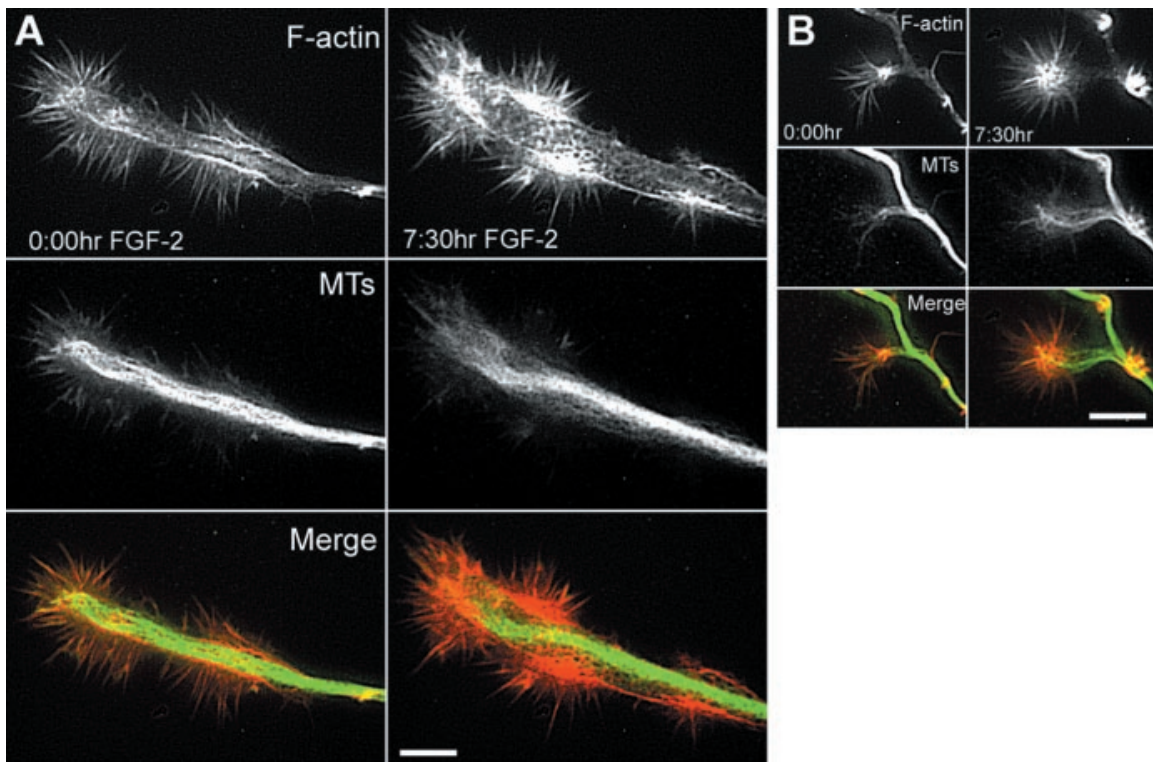

Figure 7. FGF-2 increases F-actin in growth cones and causes microtubules to splay apart. $A$, Images of a living elongated growth cone from a P3 cortical neuron coinjected with Alexa488 phalloidin (red in merged image) to label F-actin and rhodamine tubulin (green in merged image) to label MTs. FGF-2 $(10 \mathrm{ng} / \mathrm{ml})$ was added at the time that the first set of images were collected. After $7 \mathrm{hr} 30 \mathrm{~min}$, the intensity of the F-actin has greatly increased, and the microtubules are beginning to splay apart and form a loop in the growth cone (all images acquired at the same camera settings). $B$, Images of a newly formed axon branch from a neuron coinjected as in A. After $7 \mathrm{hr} 30$ min of FGF-2 application, the growth cone F-actin intensity has greatly increased as have the number of filopodia. Intense F-actin puncta have also formed in the proximal and distal axon shaft. Concomitantly, microtubules in the branch and growth cone have begun to splay apart and form a loop. Scale bars, $10 \mu \mathrm{m}$.

merizing actin filaments, attenuating microtubule dynamics, and collapsing microtubule arrays within minutes. The rapid development of axon branches by application of netrin-1 did not always involve the growth cone but could induce localized accumulation of actin filaments in filopodial extensions along the axon shaft. Importantly, polymerization of actin filaments and microtubule dynamics were necessary for axon branching to occur. Taken together, these results show that guidance factors elicit positive or inhibitory effects on axon branching by influencing the organization and dynamics of the cytoskeleton at the growth cone and the axon shaft.

\section{Regulation of axonal growth versus branching}

Although it was generally the case that cues attractive or repellent to cortical axons increased or decreased their branching, respectively, our results also suggest that axon outgrowth and axon branching are regulated independently. This is consistent with the situation in vivo in which axons stop elongating while collaterals are extending toward their targets (Bagri et al., 2003). Our studies are also consistent with other studies showing that netrin-1 increases and Sema3A decreases axon outgrowth, because in these studies only total neurite length or the longest neurite was measured. Thus if we were to combine axon length with branch length our data would also show an increase in axon outgrowth after treatment with netrin-1 and FGF-2 and a decrease in axon outgrowth after treatment with Sema3A. Interestingly, recent findings have shown that Sema3A and the Slit proteins repel cortical axons but attract or promote, respectively, the growth and branching of their dendrites (Polleux et al., 2000; Whitford et al., 2002a,b). Thus, in many situations the growth of cortical neuronal processes can be differentially regulated by a single guidance factor. Recent findings in Drosophila suggest that independent regulation of axon outgrowth and branching may be a developmental mechanism common to many species. In mushroom body neurons, it was found that progressive loss of three Rac GTPases leads first to defects in axon branching and then to guidance and finally growth (Ng et al., 2002). These results suggest that growth, guidance, and branching may be mechanistically linked but require different levels of Rac GTPase activity, which is involved in regulation of the actin cytoskeleton.

\section{Regulation of branching through changes in primary growth cones} Previous studies in cortical slices (Halloran and Kalil, 1994) and dissociated cell cultures (for review, see Kalil et al., 2000) have shown that pausing behaviors and enlargement of the primary growth cone demarcate branch points along cortical axons. These branches develop interstitially from remnants left behind on the axon by large paused growth cones. In the present study we found that effects of FGF-2 and Sema3A on axon branching are mediated by effects on the primary growth cone. Sema3A collapsed cortical growth cones, and the resulting absence of large pausing growth cones reduced branching by $\sim 50 \%$. In agreement with previous results, FGF-2 increased axon branching by inducing growth cones to pause and enlarge (Szebenyi et al., 2001). Netrin-1, however, decreased the numbers of large paused growth cones, and direct time-lapse observations showed that many branches resulting from netrin-1 treatment extended directly from the axon shaft rather than from expanded branch points. The rapid growth of branches after a few hours in netrin-1-treated cultures, in contrast to the growth of branches over several days in control and FGF-2-treated cultures, suggests that different signaling mechanisms may be involved. Time-lapse observations in living brain slices have demonstrated that branching of cortical axons in response to cues from pontine targets can occur by extension of filopodial processes directly from the axon shaft (Bastmeyer and O'Leary, 1996). Our results from bath and local application of netrin-1 also show that branches can form as filopodial extensions from the axon shaft without involvement of the primary growth cone. Recent evidence suggests that axon pruning involves diverse axon remodeling processes (Kantor and Kolodkin, 2003), including selective retraction (Bagri et al., 2003) and local degeneration (Watts et al., 2003) of axon branches. Similarly, in different contexts, axon branches may develop by different cellular mechanisms. For example, interstitial collaterals may form directly from axons shafts in pathways (Bastmeyer and O'Leary, 1996), whereas the growth of terminal axon arbors may be elaborated as branches from paused growth cones (Hall et al., 2000).

\section{Cytoskeletal reorganization in response to guidance cues}

Changes in the direction of axon growth at the growth cone and at axon branch points require reorganization of the cytoskeleton (Kalil et al., 2000; Dickson, 2002; Luo, 2002; Dent and Gertler, 2003; Dent et al., 2003). We showed previously that cortical axon branching involves changes in levels of F-actin and in microtubule dynamics on the axon shaft and at the growth cone (Dent and Kalil, 2001). 
Growth and guidance factors influence axon outgrowth at the growth cone and at axon branch points, but the precise effects of such factors on reorganization of the cytoskeleton have not been studied extensively. Local application of positive factors such as NGF and BDNF have been shown to elicit axon branching by filopodial and lamellipodial protrusions that were accompanied by an increase in F-actin and local debundling of microtubules (Gallo and Letourneau, 1998; Gibney and Zheng, 2003). These protrusions occurred directly from the axon shaft. In contrast, bath application of neurotrophin-3 (NT-3) to hippocampal neurons appeared to increase axon branching by increasing the size of large growth cones containing prominent microtubule loops (Morfini et al., 1994). In the present study, we found that the attractive guidance factors FGF-2 and netrin-1 promoted axon branching by reorganization of the cytoskeleton over different time courses and in some cases by different mechanisms. FGF-2, like NT-3, promoted axon branching by increasing actin polymerization and splaying of microtubules and in some cases the formation of microtubule loops in large paused growth cones over many hours. However, netrin-1induced branching was much more rapid and in many cases did not involve the growth cone. Nevertheless, netrin-1induced branching from the axon shaft also required actin polymerization and microtubule debundling. These results are consistent with a recent study on KIF2A, a kinesin family member that interacts directly with microtubules by inhibiting their ability to elongate and splay apart. KIF2A was shown to be required for suppressing supernumerary branches on the axons of hippocampal neurons (Homma et al., 2003). When KIF2A was knocked out, microtubules were then able to splay from the central microtubule bundle and contact the membrane, thereby inducing protrusions leading to axon branching.

The effects of inhibitory factors such as Sema3A on the cytoskeleton have been more difficult to characterize because their effects lead to growth cone collapse (Fan et al., 1993; Fritsche et al., 1999; Fournier et al., 2000; Aizawa et al., 2001). In the present study, we cultured cortical neurons on a highly adhesive substrate, poly-D-lysine, which prevented growth cone collapse that occurs on laminin, a less adhesive substrate, and allowed us to visualize dynamic changes in the cytoskeleton after application of Sema3A. Live cell imaging revealed the rapid dissolution of F-actin bundles similar to that reported for Helisoma growth cones treated with the collapsing factor, serotonin (Zhou and Cohan, 2001; Zhou

\section{$\mathbf{G}$}
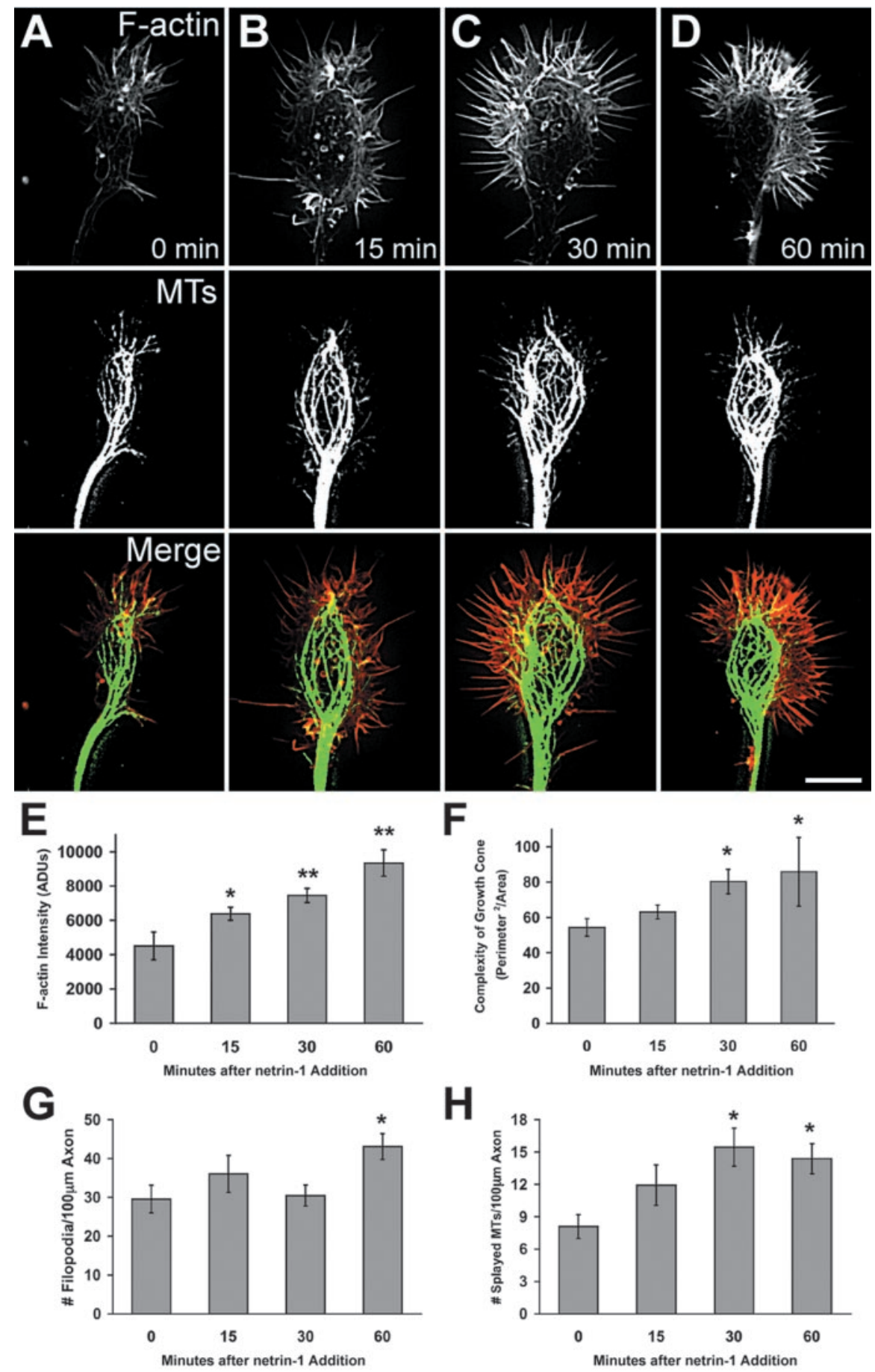

$\mathbf{F}$

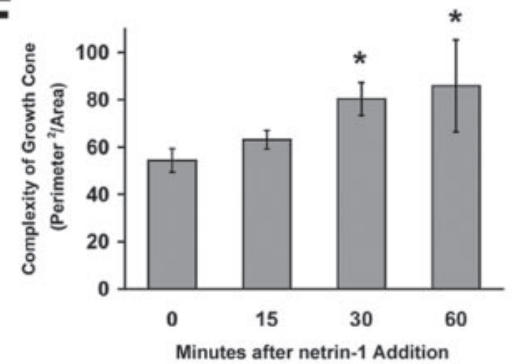

$\mathrm{H}$

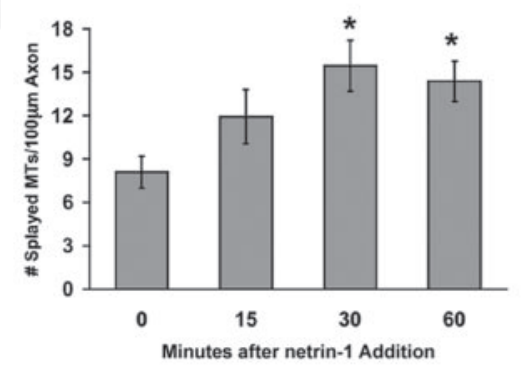

Figure 8. Netrin-1 increases F-actin, the complexity of the growth cone, and the number of filopodia and splayed microtubules along the axon shaft. $A-D$, Examples of cortical growth cones fixed and double labeled for F-actin (phalloidin stain) MTs (antityrosinated microtubule label) after 0-60 min of netrin-1 addition $(250 \mathrm{ng} / \mathrm{ml})$. E, Bar graphs showing the increase in F-actin intensity after addition of netrin-1. For all graphs, ${ }^{*} p<0.05$ and ${ }^{* *} p<0.01$; ANOVA with Dunn's post hoc tests. F, Bar graphs showing the increase in growth cone complexity over time after addition of netrin-1. $G$, Bar graphs showing increase in the number of filopodia per $100 \mu \mathrm{m}$ of axon over time after addition of netrin-1. $H$, Bar graphs showing increase in the number of individual splayed microtubules from the central microtubule bundle per $100 \mu \mathrm{m}$ of axon over time, after addition of netrin-1. Neurons were from P1 and P3 cortical cultures. All error bars are \pm SEM. Scale bar, $10 \mu \mathrm{m}$.

et al., 2002). Sema3A also diminished microtubule dynamics in the growth cone periphery. Because microtubules extend into the growth cone periphery along actin bundles (Dent and Kalil, 2001; Schaefer et al., 2002; Zhou et al., 2002), the loss of actin bundles and the maintenance of retrograde actin flow caused microtubules to 

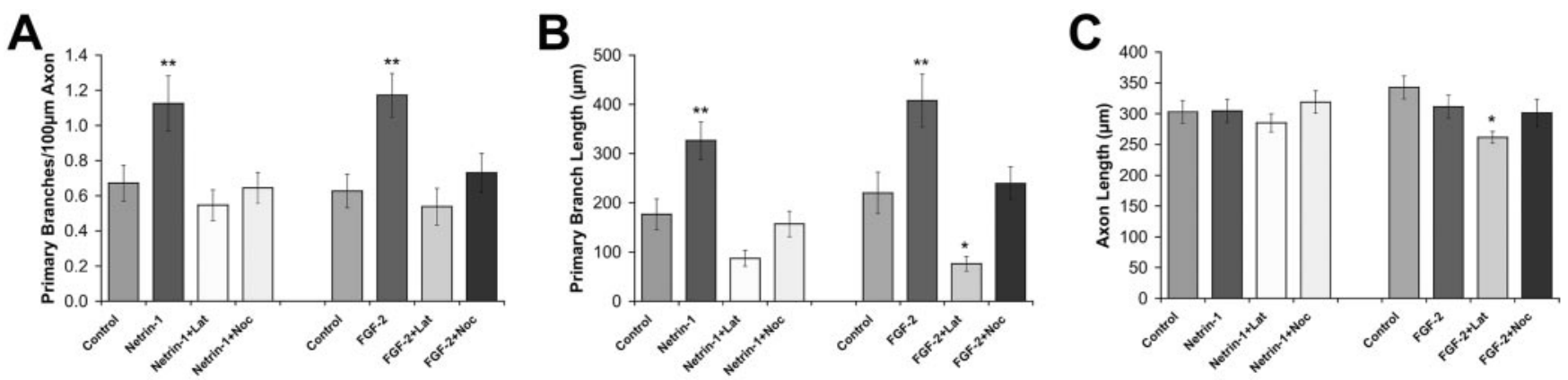

Figure 9. Treatments that attenuate microtubule and actin dynamics inhibit the increase in axon branching after addition of netrin-1 and FGF-2. A, B, Bar graphs showing that treatment ( 72 hr) of the cultures with $0.5 \mu \mathrm{m}$ latrunculin A (Lat) or $33 \mathrm{~nm}$ nocodazole (Noc) abolishes the increase in axon branch number and length induced by netrin- 1 ( $250 \mathrm{ng} / \mathrm{ml})$ or FGF-2 (10 ng/ml). Both netrin-1 and FGF-2 significantly increase branch number and length ( ${ }^{* *} p<0.01$ compared with controls; ANOVA with Dunn's post hoc test; $n>20$ neurons for all groups), and addition of latrunculin A or nocodazole significantly decreases branch number and length to control levels. Latrunculin slightly decreases FGF-2-induced branch length below control level $\left(^{*} p<0.05\right.$ compared with control; ANOVA with Dunn's post hoc test). C, Bar graphs showing that after treatments with nocodazole axon length is not changed. Treatment with latrunculin A slightly decreases axon length after treatment with FGF-2 ( ${ }^{*} p<0.05$ compared with control). All error bars are \pm SEM.

lose their exploratory abilities and consequently collapse rearward onto the central microtubule loop. Actin depolymerization and loss of microtubule dynamics eventually led to a large decrease in axon branching. This result is similar to our previous finding that latrunculin A, through similar (although nonphysiological) effects on the cytoskeleton, also decreased axon branching without affecting axon length (Dent and Kalil, 2001); however, for the first time, we have shown here that attractive and inhibitory guidance factors can regulate axon branching by opposing effects on both the actin and microtubule cytoskeleton. Given that similar cytoskeletal dynamics occur during axon guidance at the primary growth cone and at axon branch points, it will be important in future studies to elucidate differences in signaling pathways whereby guidance factors can independently regulate axon outgrowth and branching.

\section{References}

Aizawa H, Wakatsuki S, Ishii A, Moriyama K, Sasaki Y, Ohashi K, SekineAizawa Y, Sehara-Fujisawa A, Mizuno K, Goshima Y, Yahara I (2001) Phosphorylation of cofilin by LIM-kinase is necessary for semaphorin 3A-induced growth cone collapse. Nat Neurosci 4:367-373.

Bagnard D, Lohrum M, Uziel D, Puschel AW, Bolz J (1998) Semaphorins act as attractive and repulsive guidance signals during the development of cortical projections. Development 125:5043-5053.

Bagri A, Cheng HJ, Yaron A, Pleasure SJ, Tessier-Lavigne M (2003) Stereotyped pruning of long hippocampal axon branches triggered by retraction inducers of the semaphorin family. Cell 113:285-299.

Bastmeyer M, O'Leary DD (1996) Dynamics of target recognition by interstitial axon branching along developing cortical axons. J Neurosci 16:1450-1459.

Bowie JE, Young IT (1977) An analysis technique for biological shape-II. Acta Cytol 21:455-464.

Braisted JE, Catalano SM, Stimac R, Kennedy TE, Tessier-Lavigne M, Shatz CJ, O'Leary DD (2000) Netrin-1 promotes thalamic axon growth and is required for proper development of the thalamocortical projection. J Neurosci 15:5792-5801.

Campbell DS, Regan AG, Lopez JS, Tannahill D, Harris WA, Holt CE (2001) Semaphorin $3 \mathrm{~A}$ elicits stage-dependent collapse, turning, and branching in Xenopus retinal growth cones. J Neurosci 21:8538-8547.

Castellani V, Rougon G (2002) Control of semaphorin signaling. Curr Opin Neurobiol 12:532-541.

Davenport RW, Thies E, Cohen ML (1999) Neuronal growth cone collapse triggers lateral extensions along trailing axons. Nat Neurosci 2:254-259.

de la Torre JR, Hopker VH, Ming G, Poo M, Tessier-Lavigne M, HemmatiBrivanlou A, Holt CE (1997) Turning of retinal growth cones in a netrin-1 gradient mediated by the netrin receptor DCC. Neuron 19:1211-1224.

Dent EW, Gertler FB (2003) Cytoskeletal dynamics and transport in growth cone motility and axon guidance. Neuron 40:209-227.
Dent EW, Kalil K (2001) Axon branching requires interactions between dynamic microtubules and actin filaments. J Neurosci 21:9757-9769.

Dent EW, Kalil K (2003) Dynamic imaging of neuronal cytoskeleton. Methods Enzymol 361:390-407.

Dent EW, Meiri KF (1992) GAP-43 phosphorylation is dynamically regulated in individual growth cones. J Neurobiol 23:1037-1053.

Dent EW, Callaway JL, Szebenyi G, Baas PW, Kalil K (1999) Reorganization and movement of microtubules in axonal growth cones and developing interstitial branches. J Neurosci 19:8894-8908.

Dent EW, Tang F, Kalil K (2003) Axon guidance by growth cones and branches: common cytoskeletal and signaling mechanisms. Neuroscientist 9:343-353.

Dickson BJ (2002) Molecular mechanisms of axon guidance. Science 298:1959-1964.

Fan J, Mansfield SG, Redmond T, Gordon-Weeks PR, Raper JA (1993) The organization of F-actin and microtubules in growth cones exposed to a brain-derived collapsing factor. J Cell Biol 121:867-878.

Forcet C, Stein E, Pays P, Corset V, Llambl F, Tessier-Lavigne M, Mehlen P (2002) Netrin-1 mediated axon outgrowth requires deleted in colorectal cancer-dependent MAPK activation. Nature 417:443-447.

Fournier AE, Nakamura F, Kawamoto S, Goshima Y, Kalb RG, Strittmatter SM (2000) Semaphorin3A enhances endocytosis at sites of receptor-Factin colocalization during growth cone collapse. J Cell Biol 149:411-422.

Fritsche J, Reber BF, Schindelholz B, Bandtlow CE (1999) Differential cytoskeletal changes during growth cone collapse in response to hSema III and thrombin. Mol Cell Neurosci 14:398-418.

Gallo G, Letourneau PC (1998) Localized sources of neurotrophins initiate axon collateral sprouting. J Neurosci 18:5403-5414.

Gallo G, Letourneau PC (1999) Different contributions of microtubule dynamics and transport to the growth of axons and collateral sprouts. J Neurosci 19:3860-3873.

Gibney J, Zheng JQ (2003) Cytoskeletal dynamics underlying collateral membrane protrusions induced by neurotrophins in cultured Xenopus embryonic neurons. J Neurobiol 54:393-405.

Hall AC, Lucas FR, Salinas PC (2000) Axonal remodeling and synaptic differentiation in the cerebellum is regulated by WNT-7a signaling. Cell 100:525-535.

Halloran MC, Kalil K (1994) Dynamic behaviors of growth cones extending in the corpus callosum of living cortical brain slices observed with video microscopy. J Neurosci 14:2161-2177.

Homma N, Takei Y, Tanaka Y, Nakata T, Terada S, Kikkawa M, Noda Y, Hirokawa N (2003) Kinesin superfamily protein 2A (KIF2A) functions in suppression of collateral branch extension. Cell 114:229-239.

Hopker VH, Shewan D, Tessier-Lavigne M, Poo M, Holt C (1999) Growthcone attraction to netrin-1 is converted to repulsion by laminin-1. Nature 401:69-73.

Kalil K, Szebenyi G, Dent EW (2000) Common mechanisms underlying growth cone guidance and axon branching. J Neurobiol 44:145-158.

Kantor DB, Kolodkin AL (2003) Curbing the excesses of youth: molecular insights into axonal pruning. Neuron 38:849-852. 
Kennedy TE, Tessier-Lavigne M (1995) Guidance and induction of branch formation in developing axons by target-derived diffusible factors. Curr Opin Neurobiol 5:83-90.

Kuang RZ, Kalil K (1994) Development of specificity in corticospinal connections by axon collaterals branching selectively into appropriate spinal targets. J Comp Neurol 344:270-282.

Luo L (2002) Actin cytoskeleton regulation in neuronal morphogenesis and structural plasticity. Annu Rev Cell Dev Biol 18:601-635.

Manitt C, Kennedy TE (2002) Where the rubber meets the road: netrin expression and function in developing and adult nervous systems. Prog Brain Res 137:425-442.

Metin C, Deleglise D, Serafini T, Kennedy TE, Tessier-Lavigne M (1997) A role for netrin-1 in the guidance of cortical efferents. Development 124: 5063-5074.

Ming GL, Wong ST, Henley J, Yuan XB, Song HJ, Spitzer NC, Poo MM (2002) Adaptation in the chemotactic guidance of nerve growth cones. Nature 417:411-418.

Morfini G, DiTella MC, Feiguin F, Carri N, Caceres A (1994) Neurotrophin-3 enhances neurite outgrowth in cultured hippocampal pyramidal neurons. J Neurosci Res 39:219-232.

Ng J, Nardine T, Harms M, Tzu J, Goldstein A, Sun Y, Dietzl G, Dickson BJ, Luo L (2002) Rac GTPases control axon growth, guidance and branching. Nature 416:442-447.

O'Leary DD, Bicknese AR, De Carlos JA, Heffner CD, Koester SE, Kutka LJ, Terashima T (1990) Target selection by cortical axons: alternative mechanisms to establish axonal connections in the developing brain. Cold Spring Harb Symp Quant Biol 55:453-468.

Pasterkamp RJ, Kolodkin AL (2003) Semaphorin junction: making tracks toward neural connectivity. Curr Opin Neurobiol 13:79-89.

Polleux F, Giger RJ, Ginty DD, Kolodkin AL, Ghosh A (1998) Patterning of cortical efferent projections by semaphorin-neuropilin interactions. Science 282:1904-1906.

Polleux F, Morrow T, Ghosh A (2000) Semaphorin 3A is a chemoattractant for cortical apical dendrites. Nature 404:567-573.

Richards LJ, Koester SE, Tuttle R, O’Leary DD (1997) Directed growth of early cortical axons is influenced by a chemoattractant released from an intermediate target. J Neurosci 17:2445-2458.
Schaefer AW, Kabir N, Forscher P (2002) Filopodia and actin arcs guide the assembly and transport of two populations of microtubules with unique dynamic parameters in neuronal growth cones. J Cell Biol 158:139-152.

Serafini T, Colamarino SA, Leonardo ED, Wang H, Beddington R, Skarnes WC, Tessier-Lavigne M (1996) Netrin-1 is required for commissural axon guidance in the developing vertebrate nervous system. Cell 87:1001-1014.

Spector I, Shochet NR, Blasberger D, Kashman Y (1989) Latrunculinsnovel marine macrolides that disrupt microfilament organization and affect cell growth: I. Comparison with cytochalasin D. Cell Motil Cytoskeleton 13:127-144.

Szebenyi G, Callaway JL, Dent EW, Kalil K (1998) Interstitial branches develop from active regions of the axon demarcated by the primary growth cone during pausing behaviors. J Neurosci 18:7930-7940.

Szebenyi G, Dent EW, Callaway JL, Seys C, Lueth H, Kalil K (2001) Fibroblast growth factor-2 promotes axon branching of cortical neurons by influencing morphology and behavior of the primary growth cone. J Neurosci 21:3932-3941.

Vasquez RJ, Howell B, Yvon AM, Wadsworth P, Cassimeris L (1997) Nanomolar concentrations of nocodazole alter microtubule dynamic instability in vivo and in vitro. Mol Biol Cell 8:973-985.

Watts RJ, Hoopfer ED, Luo L (2003) Axon pruning during Drosophila metamorphosis: evidence for local degeneration and requirement of the ubiquitin-proteasome system. Neuron 38:871-885.

Whitford KL, Dijkhuizen P, Polleux F, Ghosh A (2002a) Molecular control of cortical dendrite development. Annu Rev Neurosci 25:127-149.

Whitford KL, Marillat V, Stein E, Goodman CS, Tessier-Lavigne M, Chedotal A, Ghosh A (2002b) Regulation of cortical dendrite development by Slit-Robo interactions. Neuron 33:47-61.

Zhou FQ, Cohan CS (2001) Growth cone collapse through coincident loss of actin bundles and leading edge actin without actin depolymerization. J Cell Biol 153:1071-1084.

Zhou FQ, Waterman-Storer CM, Cohan CS (2002) Focal loss of actin bundles causes microtubule redistribution and growth cone turning. J Cell Biol 157:839-849. 